Artículo

\title{
Uso de diferentes proporciones de led rojos y azules para mejorar el crecimiento de Lilium spp.
}

\author{
Silvia Flores-Pérez ${ }^{1 \S}$ \\ Ana María Castillo-González ${ }^{1}$ \\ Luis Alonso Valdez-Aguilar ${ }^{2}$ \\ Edilberto Avítia-García ${ }^{1}$ \\ ${ }^{1}$ Instituto de Horticultura-Universidad Autónoma Chapingo. Carretera México-Texcoco km 36.5, Chapingo, \\ Estado de México. CP. 56230. (anasofiacasg@hotmail.com; avidil_ag@ hotmail.com). ${ }^{2}$ Departamento de \\ Horticultura-Universidad Autónoma Agraria Antonio Narro. Calzada Antonio Narro 1923, Saltillo, \\ Coahuila. CP. 25315. (luisalonso.valdez@uaaan.mx). \\ ${ }^{\S}$ Autora para correspondencia: flopersilvia@ hotmail.com.
}

\section{Resumen}

La combinación de diodos emisores de luz (LEDs) rojos y azules es una fuente de luz efectiva para el crecimiento y desarrollo de las plantas. Diversas especies del género Lilium son valoradas como flores de corte, pero la información que se tiene del efecto de la calidad de la luz sobre su crecimiento es aún muy escasa y es necesario determinar el espectro de luz óptimo que permita obtener características deseables. El objetivo de este trabajo fue evaluar el crecimiento de Lilium spp. 'Corvara', con iluminación LED. La proporción de luces LED roja y azul en cada tratamiento fue: 20:80 (R4B); 40:60 (2R3B); 60:40 (3R2B); 80:20 (4RB) y el testigo (W) con $100 \%$ de luz blanca. Todos los tratamientos tuvieron una radiación fotosintéticamente activa de $90 \pm 10 \mu \mathrm{mol} \mathrm{m}^{-2} \mathrm{~s}^{-1}$ durante un fotoperiodo de $14 \mathrm{~h}$. El trabajo se realizó en Chapingo, México en 2019 y 2020. Los resultados mostraron que con la iluminación del tratamiento 2R3B se registró la menor cantidad de días a madurez de cosecha (91 días) y las plantas de menor altura $(83 \mathrm{~cm})$. Respecto al testigo se lograron incrementos en las siguientes variables: con el tratamiento R4B, área foliar 26\% y color de tépalos 13\% en la variable Croma; con 3R2B la vida en florero $6 \%$, con $4 \mathrm{RB}$, altura de plantas $21 \%$, diámetro de flor $5 \%$ y el número de días a madurez fue 16. Se concluye que la proporción de la luz roja y azul modifica el crecimiento y desarrollo de las plantas de Lilium spp. 'Corvara'.

Palabras clave: flores de corte, iluminación suplementaria, LED’s, vida en florero.

Recibido: enero de 2021

Aceptado: mayo de 2021 


\section{Introducción}

A nivel mundial el Lilium ocupa el décimo primer lugar en demanda dentro de las ornamentales, lo cual se atribuye a la diversidad de colores y la disponibilidad de la flor durante todo el año (García y Companioni, 2018). Estadísticas del Servicio de Información Agroalimentaria y Pesquera (SIAP, 2020) indican que en México en 270 ha, se alcanzó una producción de 735472 gruesas.

La respuesta de las plantas al espectro de luz recibido está determinada por la acción de los distintos fotorreceptores, de acuerdo con Xie et al. (2019) estos pueden ser agrupados de acuerdo con la región del espectro electromagnético que detecten: los fitocromos detectan el rojo (600 a $700 \mathrm{~nm})$ y rojo lejano (700 a $750 \mathrm{~nm}$ ) en una relación dinámica de foto equilibrio y los criptocromos y fototropinas tienen respuesta a la luz azul de 350 a $500 \mathrm{~nm}$ (Fantini et al., 2019).

Los diodos emisores de luz (LEDs) son la primera fuente de luz que permite la selección de longitudes de onda específicas en el espectro de iluminación que coinciden con la absorbancia de los fotorreceptores de las plantas e impacta en procesos vitales específicos (Morrow, 2008). La cantidad de horas luz por día impacta directamente en la floración de las plantas; en este aspecto las plantas se pueden dividir en tres categorías según la duración del día que se requiere para desencadenar la floración: plantas de día corto, plantas de día neutro y plantas de día largo. Lilium se clasifica como de día largo facultativo; es decir, que puede florecer bajo un rango amplio de longitud del día, pero la floración es acelerada o aumentada en días largos (Dole y Wilkins, 2005).

La iluminación con LEDs tiene un amplio potencial para el cultivo de plantas ornamentales; se ha utilizado como fuente de luz suplementaria para propagar plántulas y esquejes de calibrachoa (Calibrachoa hibrida), en la cual el desarrollo de raíces y brotes fue mayor con una combinación de LEDs blancos y azules (Olschowski et al., 2016), es una forma no contaminante de controlar la altura de las plantas (Bergstrand et al., 2016), se puede reducir la extensión del tallo y dar como resultado plantas más compactas en impatiens (Impatiens walleriana), petunia (Petunia hibrida) y salvia (Salvia officinalis) con una mayor proporción de luz azul que complementa la luz roja (Wollaeger y Runkle, 2013), en plantas de celosía (Celosia argentea), impatiens, petunia, caléndula (Calendula officinalis), salvia y pensamiento (Viola odorata) cultivadas bajo proporciones de luz roja: azul de 85:15 y 70:30.

Se obtuvieron plantas más compactas, con un diámetro de tallo más grande y un mayor contenido de clorofila que las plantas cultivadas con lámparas de alta presión de sodio (Randall y López, 2014), las luces LED de rojo lejano inhiben la floración de crisantemo (Chrysanthemum morifolium) y la promueven en boca de dragón (Antirrhinum majus) y petunia, mientras que con altas intensidades de luz azul se induce la floración en coreopsis (Coreopsis grandiflora) y rudbeckia (Rudbeckia hirta L.), esto permite a los cultivadores controlar la floración de acuerdo con la demanda del mercado (Meng y Runkle, 2016).

Las respuestas específicas de las plantas al espectro de luz a veces pueden ser predecibles en base a investigaciones publicadas; sin embargo, la reacción general de las plantas es difícil de prever debido a la complicada interacción de muchas respuestas internas diferentes, ocasionadas por la acción de los distintos fotorreceptores (Wollaeger y Runkle, 2013), que tienen efecto en el metabolismo primario (producción de aminoácidos, nucleótidos, azúcares y lípidos) y secundario (terpenos, fenoles y alcaloides) (Darko et al., 2014). 
La combinación de LEDs rojos y azules es una fuente de luz efectiva para el crecimiento y desarrollo de las plantas; sin embargo, la respuesta a la calidad de luz cambia entre especies e incluso entre cultivares y su etapa de desarrollo (Bayat et al., 2018). El objetivo de este trabajo fue evaluar el efecto de la iluminación s LED en el cultivo de Lilium spp. 'Corvara' para encontrar la proporción de luz LED que permita tener características deseables y un mayor control del crecimiento, floración y vida en florero con iluminación proporcionada por lámparas LEDs con diferentes proporciones de luz azul (B), rojo (R) y blanca (W) como testigo, la hipótesis es que solo con la combinación de luz roja y azul es posible tener un crecimiento normal.

\section{Materiales y métodos}

\section{Localización}

El estudio se realizó en un cuarto de crecimiento hecho de madera construido dentro de un invernadero con cubierta de cristal del Instituto de Horticultura de la Universidad Autónoma Chapingo, ubicado a una altitud de $2240 \mathrm{~m}$ y a $19^{\circ} 29^{\prime}$ de latitud norte y $38^{\circ} 53^{\prime}$ de longitud oeste Servicio Meteorológico Nacional (2021). El cuarto de crecimiento se dividió en cinco compartimientos con una superficie de $0.91 \mathrm{~m}^{2}$ cada uno. La humedad relativa y la temperatura ambiental (Cuadro 1) dentro del cuarto de crecimiento fueron monitoreados con un dato logger Perfect Prime ${ }^{\circledR}$ (Japón).

Cuadro 1. Temperatura y humedad relativa dentro del cuarto de crecimiento durante el ciclo de cultivo de Lilium spp 'Corvara' con iluminación suplementaria LED.

\begin{tabular}{|c|c|c|c|c|c|c|}
\hline \multirow{2}{*}{ Mes } & \multicolumn{2}{|c|}{ Temperatura diurna $\left({ }^{\circ} \mathrm{C}\right)$} & \multicolumn{2}{|c|}{ Temperatura nocturna $\left({ }^{\circ} \mathrm{C}\right)$} & \multicolumn{2}{|c|}{ Humedad relativa $(\%)$} \\
\hline & Mín & Máx & Mín & Máx & Mín & Máx \\
\hline Octubre & 22.8 & 27.2 & 12 & 19.6 & 38.5 & 65.4 \\
\hline Noviembre & 19.1 & 24.5 & 9.1 & 16.8 & 45.4 & 68.9 \\
\hline Diciembre & 13 & 23.4 & 7.4 & 14.1 & 44 & 70.1 \\
\hline Enero & 11.6 & 23.3 & 6.9 & 13 & 42.1 & 58.9 \\
\hline Febrero & 17.7 & 24.8 & 9 & 14.3 & 46.5 & 67.6 \\
\hline
\end{tabular}

\section{Material vegetal y sustrato}

Se usaron bulbos de 20 a $22 \mathrm{~cm}$ de perímetro de Lilium spp. 'Corvara' del tipo oriental (flor con tépalos color rosa con borde blanco), los bulbos se lavaron con agua, se desinfectaron con Tecto ${ }^{\circledR}$ $\left(2 \mathrm{~g} \mathrm{~L}^{-1}\right)$ y se colocaron a $7 \mathrm{~cm}$ de profundidad a partir del ápice del bulbo, en bolsas de plástico negro de $8 \mathrm{~L}$ el 29 de octubre de 2019. Como sustrato se utilizó tezontle rojo con diámetro de partícula de 2 a $3 \mathrm{~mm}$, debido a su disponibilidad, bajo costo de adquisición, capacidad de retención de agua y pH neutro (Ojodeagua et al., 2008).

\section{Solución nutritiva}

Se utilizó la solución de Steiner (1961) al 75\% $\left(\mathrm{NO}_{3}{ }^{-}, \mathrm{H}_{2} \mathrm{PO}_{4}{ }^{-}, \mathrm{SO}_{4}, \mathrm{~K}^{+}, \mathrm{Ca}^{2+}\right.$ y $\mathrm{Mg}^{2+}$, con una concentración de 9, 1, 5, 5, 7 y 3 meq $\mathrm{L}^{-1}$, respectivamente) durante todo el ciclo de cultivo. Para abastecer los micronutrimentos se utilizaron $0.025 \mathrm{~g} \mathrm{~L}^{-1}$ de Tradecorp ${ }^{\circledR}$, el cual contiene una mezcla 
de fertilizantes quelados con EDTA, la cual contiene: hierro $7.5 \%$, manganeso $3.5 \%$, zinc $0.7 \%$, boro $0.65 \%$, cobre $0.28 \%$ y molibdeno $0.26 \%$. La solución nutritiva fue preparada con agua potable considerando las propiedades químicas para su formulación. El pH de la solución nutritiva se ajustó a 5.7 con ácido sulfúrico; la conductividad eléctrica fue de $2.1 \mathrm{dS} \mathrm{m} \mathrm{m}^{-1}$. Cada planta se regó todos los días de forma manual con $200 \mathrm{ml}$ los primeros 15 días, posteriormente se aplicaron $350 \mathrm{ml}$.

\section{Tratamientos}

En cada compartimiento del cuarto de cultivo se ubicó un tratamiento, cada uno consistió en lámparas de 200 Watts, integrada por 20 LEDs monocromáticos TIANLAI $^{\circledR}$ (China). La proporción en \% de luces LED roja y azul en cada tratamiento fue: 20:80 (R4B); 40:60 (2R3B); 60:40 (3R2B); 80:20 (4RB) y el testigo (W) con 100\% de luz blanca. Las lámparas se subieron a medida que las plantas iban creciendo para que la luz incidiera sobre los ápices con una radiación fotosintéticamente activa (PAR) de $90 \pm 10 \mu \mathrm{mol} \mathrm{m}^{-2} \mathrm{~s}^{-1}$, la PAR se determinó con un medidor cuántico hidrofarm ${ }^{\circledR}$ (CA, USA).

Las longitudes de onda de los LEDs fueron: azul de 450 a $480 \mathrm{~nm}$ con un máximo en $465 \mathrm{~nm}$, roja de 620 a $650 \mathrm{~nm}$ con un máximo en $635 \mathrm{~nm}$ y blanco con un espectro de luz de 400 a $700 \mathrm{~nm}$, las cuales estaban dentro del rango indicado por Deram et al. (2014) quienes señalaron que la combinación de luz azul $(425-490 \mathrm{~nm})$ y luz roja $(610-700 \mathrm{~nm})$ son los mejores espectros de luz para la fotosíntesis de las plantas. Las longitudes de onda emitidas por los LEDs se midieron con un espectroradiómetro (CS-2000, Konica Minolta ${ }^{\circledR}$, Japón). Las lámparas se mantuvieron encendidas de forma continua de 7 a $21 \mathrm{~h}$, el encendido y apagado se programó con un temporizador digital Voltech TEM-8 (China).

\section{Diseño experimental}

Fue un diseño completamente al azar, cinco tratamientos y nueve repeticiones, la unidad experimental consistió en una planta por bolsa, con un total de 45 unidades experimentales.

\section{Variables evaluadas}

Los tallos florales se cortaron del 29 de enero al 12 de febrero de 2020, cuando la primera flor de cada planta estaba completamente abierta. De cada tratamiento se tomaron las primeras cuatro plantas para evaluar la vida en florero, a cada tallo se le cortaron las hojas excepto las de la inflorescencia y de esas hojas se tomaron las muestras para determinar la concentración de azúcares solubles totales y pigmentos fotosintéticos, por esto, las variables mencionadas tienen cuatro repeticiones. En las cinco plantas restantes de cada tratamiento se evaluó el área foliar y la densidad estomática, como se describe posteriormente. Las demás variables se midieron en nueve repeticiones por tratamiento. La determinación de las variables evaluadas se describe a continuación.

\section{Días a madurez de cosecha y duración de la etapa vegetativa (días)}

Los días a madurez se contaron a partir de la plantación de los bulbos hasta el corte de los tallos florales; la etapa vegetativa se contó a partir de la plantación de los bulbos hasta que fue visible el primer botón floral en cada planta. 


\section{Altura de planta (cm)}

Se midió con una cinta métrica desde la base del tallo hasta el ápice de los botones florales.

\section{Peso fresco y seco de la parte aérea $(\mathrm{g})$}

La planta se separó en parte aérea, bulbo y raíces, se registró el peso fresco con una balanza digital Ohaus ${ }^{\circledR}$ modelo Scout Pro (NJ, USA), después las muestras se lavaron con agua destilada, se secaron en un horno con aire circulante Binder ${ }^{\circledR}$ (Berlín, Alemania) a $65^{\circ} \mathrm{C}$ por $48 \mathrm{~h}$ y se volvieron a pesar las muestras.

\section{Diámetro del tallo (mm)}

Se midió debajo de la inflorescencia con un vernier digital Trupper ${ }^{\circledR}$ (China).

\section{Área foliar $\left(\mathbf{c m}^{2}\right)$}

Se separaron todas las hojas del tallo, excepto las hojas de la inflorescencia, se evaluó con un integrador de área foliar Li-cor 3100 (NB, USA).

\section{Diámetro de la flor $(\mathrm{cm})$}

Se midió con cinta métrica entre los ápices de los pétalos de la primera flor completamente abierta de cada planta.

\section{Número, longitud (cm) y diámetro de botones (mm)}

Se contaron los botones florales de cada planta al corte los tallos florales; se midió en cada planta la longitud del primer botón cerrado que presentó el color rosa del cultivar, desde su base hasta la parte apical, en el mismo botón se midió el diámetro por la parte más ancha con un vernier digital Trupper $^{\circledR}$ (China).

\section{Color (L, H, C)}

Se determinó en tres puntos diferentes de la parte media de los tépalos de la primera flor abierta en cada planta, con un espectrofotómetro X- Rite Inc. (MI, USA), se obtuvieron los parámetros: L que es brillantez o luminosidad, cuyos valores van de 0 a 100, donde 0 representa el color negro y 100 el blanco, $\mathrm{H}$ o ángulo de tono (hue), el cual se define como el ángulo entre la hipotenusa y $0^{\circ}$ con el eje (verde azul a rojo púrpura), C o pureza de color (croma), el cual reporta el índice de saturación, estos valores forman el modelo cromático usado para describir todos los colores que puede percibir el ojo humano (Jakopic et al., 2007).

\section{Densidad estomática (estomas $\mathbf{m m}^{2}$ )}

En una hoja por planta, en el envés de hojas basales (a $15 \mathrm{~cm}$ de altura desde la base del tallo), de la parte media (a $35 \mathrm{~cm}$ de altura) y apicales (a $60 \mathrm{~cm}$ de altura), se aplicaron dos capas de barníz transparente en cinco ubicaciones al lado de la nervadura central de cada hoja, se dejaron secar, se 
cortaron las plantas y se retiraron de las hojas las capas de barníz con la epidermis (negativo de la impresión), se colocaron en portaobjetos y con un microscopio óptico Carl Zeiss ${ }^{\circledR}$ (Alemania), se contó el número de estomas en cinco campos visuales (10x) por cada portaobjeto.

\section{Pigmentos fotosintéticos (mg $\mathrm{g}^{-1}$ de peso fresco)}

Se determinaron las concentraciones de clorofila a, b, clorofila total y carotenoides cuando inició la floración, de los 36 a los 46 días después de la plantación y después del corte de los tallos florales como lo indica la AOAC (1980) y la técnica descrita por Witham et al. (1971), para lo cual se utilizaron dos hojas de reciente maduración por planta. Se leyó la absorbancia a 663, 645 y $470 \mathrm{~nm}$ en un espectrofotómetro Thermo Spectronic ${ }^{\circledR}$, modelo Genesys 10 UV (WI, USA).

\section{Azúcares solubles totales ( $\mathrm{mg} \mathrm{g}^{-1}$ de peso fresco)}

Se evaluaron en hojas al comienzo de la floración y en hojas, bulbos y raíces al momento del corte de los tallos florales, con el método de antrona descrito por Witham et al. (1971), se pesaron $2 \mathrm{~g}$ de la mezcla de tres hojas de reciente maduración, $2 \mathrm{~g}$ de las escamas de los bulbos y $2 \mathrm{~g}$ de la parte media de las raíces. Las lecturas se realizaron a $600 \mathrm{~nm}$ con un espectrofotómetro Thermo Spectronic ${ }^{\circledR}$, modelo Genesys 10 UV (WI, USA). La concentración de azúcares se calculó a partir de una curva patrón que contenía hasta $250 \mu \mathrm{g}$ de glucosa $\mathrm{ml}^{-1}$.

\section{Vida en florero (días) y consumo de agua en postcosecha (ml)}

Se contó desde el corte de las plantas hasta que 50\% de las flores de cada tallo presentó síntomas de senescencia. Los tallos florales se dejaron a $70 \mathrm{~cm}$ de altura cortando la base en diagonal y se eliminó el follaje inferior, cada tallo se colocó en una probeta de $1 \mathrm{~L}$ de capacidad con $500 \mathrm{ml}$ de agua corriente, se tuvo una temperatura promedio de $18.5^{\circ} \mathrm{C}$ y humedad relativa fue de $41.7 \%$. El consumo de agua por evaporación y transpiración se determinó todos los días en cada contenedor por diferencia entre el volumen inicial y el volumen final, reponiendo el agua consumida.

\section{Análisis de datos}

Se realizó un análisis de varianza, la comparación de medias de Tukey $(p<0.05)$ y el análisis de correlación de Pearson con el programa estadístico Statistical Analysis Sistem (SAS) versión 9 (SAS Institute Inc., 2002). Las gráficas se realizaron en el programa Sigmaplot.

\section{Resultados y discusión}

\section{Variables de crecimiento y floración}

Las plantas de Lilium oriental 'Corvara' mostraron diferencias significativas entre tratamientos ( $p<$ 0.05), en las variables días a madurez de cosecha, duración de la etapa vegetativa, altura de planta, diámetro de tallo, pesos fresco y seco de la parte aérea, diámetro de flor y longitud de botón el tratamiento $4 \mathrm{RB}$ ( $80 \%$ de luz roja $+20 \%$ de luz azul) registró los valores máximos (Cuadro 2 y 3 ). Aunque con ese tratamiento se favoreció el alargamiento de los entrenudos, aumentó la duración del ciclo de cultivo en 15 días respecto al control. 
Cuadro 2. Efecto de la luz suplementaria LED roja, azul, blanca y sus combinaciones sobre las variables de crecimiento de Lilium spp. 'Corvara'.

\begin{tabular}{cccccccc}
\hline & $\begin{array}{c}\text { Días a } \\
\text { Tadurez de } \\
\text { cosecha } \\
\text { (días) }\end{array}$ & $\begin{array}{c}\text { Duración de la } \\
\text { etapa } \\
\text { vegetativa } \\
\text { (días) }\end{array}$ & $\begin{array}{c}\text { Altura } \\
(\mathrm{cm})\end{array}$ & $\begin{array}{c}\text { Diámetro de } \\
\text { tallo }(\mathrm{mm})\end{array}$ & $\begin{array}{c}\text { Peso } \\
\text { fresco } \\
\text { parte } \\
\text { aérea }(\mathrm{g})\end{array}$ & $\begin{array}{c}\text { Peso } \\
\text { seco } \\
\text { partea }(\mathrm{g})\end{array}$ & $\begin{array}{c}\text { Área } \\
\text { foliar por } \\
\text { planta } \\
\left(\mathrm{cm}^{2}\right)\end{array}$ \\
\hline R4B & $100.2 \mathrm{~b}$ & $35.9 \mathrm{c}$ & $88.1 \mathrm{~b}$ & $9.4 \mathrm{~b}$ & $199.6 \mathrm{ab}$ & $25.7 \mathrm{ab}$ & $1161 \mathrm{a}$ \\
2R3B & $90.9 \mathrm{~d}$ & $38.2 \mathrm{~b}$ & $83.2 \mathrm{~b}$ & $9.5 \mathrm{~b}$ & $166.3 \mathrm{~b}$ & $22.1 \mathrm{~b}$ & $1045 \mathrm{ab}$ \\
3R2B & $97.7 \mathrm{c}$ & $36.2 \mathrm{bc}$ & $85.2 \mathrm{~b}$ & $9.5 \mathrm{~b}$ & $196.3 \mathrm{ab}$ & $22.8 \mathrm{~b}$ & $1044 \mathrm{ab}$ \\
4RB & $106.9 \mathrm{a}$ & $46 \mathrm{a}$ & $101 \mathrm{a}$ & $10.5 \mathrm{a}$ & $238.6 \mathrm{a}$ & $28.6 \mathrm{a}$ & $1046 \mathrm{ab}$ \\
W & $91.4 \mathrm{~d}$ & $37.9 \mathrm{bc}$ & $83.4 \mathrm{~b}$ & $9.7 \mathrm{ab}$ & $216.5 \mathrm{ab}$ & $24 \mathrm{~b}$ & $907 \mathrm{~b}$ \\
CV $(\%)$ & 1.5 & 4 & 8.6 & 6.9 & 19.1 & 11.8 & 7.5 \\
DSH $(0.05)$ & 2 & 2.1 & 10.3 & 0.9 & 52.2 & 3.9 & 148 \\
\hline
\end{tabular}

Valores en la misma columna seguidos de la misma letra no son estadísticamente diferentes (Tukey, $p \leq 0.05$ ); $\mathrm{CV}=$ coeficiente de variación; $\mathrm{DSH}=$ diferencia significativa honesta. $\mathrm{R}=$ luz roja; $\mathrm{B}=\mathrm{azul} ; \mathrm{y} \mathrm{W}=$ blanca; $\mathrm{R} 4 \mathrm{~B}=\mathrm{R} 20 \%+$ B 80\%, 2R3B: R 40\% + B 60\%, 3R2B: R 60\% + B 40\%, 4RB: R 80\% + B 20\% y W=100\% de luz blanca.

Cuadro 3. Efecto de la luz suplementaria LED roja, azul, blanca y sus combinaciones sobre las variables de floración en plantas de Lillium spp. 'Corvara'.

\begin{tabular}{cccccccc}
\hline \multirow{2}{*}{ Tratamiento } & \multirow{2}{*}{$\begin{array}{c}\text { Diámetro de } \\
\text { flor }(\mathrm{cm})\end{array}$} & $\begin{array}{c}\text { Núm. de } \\
\text { botones }\end{array}$ & $\begin{array}{c}\text { Longitud de } \\
\text { botón }(\mathrm{cm})\end{array}$ & $\begin{array}{c}\text { Diámetro de } \\
\text { botón }(\mathrm{mm})\end{array}$ & \multicolumn{4}{c}{ Color de tépalos } \\
\hline R4B & $28.4 \mathrm{ab}$ & $5.8 \mathrm{~ns}$ & $14.3 \mathrm{ab}$ & $41.5 \mathrm{~ns}$ & $51.7 \mathrm{c}$ & $24.3 \mathrm{a}$ & $348.8 \mathrm{~ns}$ \\
2R3B & $25.3 \mathrm{~b}$ & 5.5 & $12.8 \mathrm{~b}$ & 38.9 & $55.2 \mathrm{bc}$ & $23.7 \mathrm{a}$ & 349.4 \\
3R2B & $28.1 \mathrm{ab}$ & 4.6 & $14.5 \mathrm{a}$ & 42.9 & $57.4 \mathrm{~b}$ & $21.7 \mathrm{ab}$ & 352.7 \\
4RB & $29.9 \mathrm{a}$ & 5.8 & $14.6 \mathrm{a}$ & 38 & $66.3 \mathrm{a}$ & $16.2 \mathrm{~b}$ & 349.5 \\
$\mathrm{~W}$ & $28.5 \mathrm{ab}$ & 5.7 & $14.8 \mathrm{a}$ & 42.3 & $55.4 \mathrm{bc}$ & $21.5 \mathrm{ab}$ & 347.6 \\
$\mathrm{CV}(\%)$ & 9 & 19.4 & 8.1 & 9.5 & 7.3 & 24.2 & 1.8 \\
DSH $(0.05)$ & 3.4 & 1.43 & 1.5 & 5.2 & 5.6 & 7 & 8.4 \\
\hline
\end{tabular}

Valores en la misma columna seguidos de la misma letra no son estadísticamente diferentes (Tukey, $p \leq 0.05$ ); $\mathrm{CV}=$ coeficiente de variación; $\mathrm{DSH}=$ diferencia significativa honesta; $\mathrm{ns}=$ no significativo; $\mathrm{L}=$ brillantez o luminosidad; $\mathrm{C}=$ croma o pureza de color; $\mathrm{H}=$ hue o ángulo de tono; $\mathrm{R}=$ luz roja; $\mathrm{B}=$ azul; $\mathrm{W}=$ blanca; $\mathrm{R} 4 \mathrm{~B}=\mathrm{R} 20 \%+\mathrm{B} 80 \%$, 2R3B: R 40\% + B 60\%, 3R2B: R 60\% + B 40\%, 4RB: R 80\% + B 2\% y W: $100 \%$ de luz blanca.

Al momento del corte la altura promedio de los tallos del tratamiento 4RB fue de $101 \mathrm{~cm}$, esto es importante ya que las normas de calidad para la comercialización de esta flor de corte, se basan en la longitud de los tallos y el número de botones, un ejemplo es Coxflor ${ }^{\circledR}$ (empresa proveedora de bulbos para producción de flores), marca una clasificación de calidad para la comercialización de Lilium: categoría plus 4 a 6 botones por tallo y una altura de $90-110 \mathrm{~cm}$ (exportación), 2 a 3 botones por tallo con una altura de 70 a $90 \mathrm{~cm}$ y media de 1 a 2 botones por tallo con una altura de $70 \mathrm{~cm}$. 
Con base en lo anterior, sólo las plantas cultivadas con el tratamiento 4RB entrarían en la categoría plus, las demás en la categoría exportación. Las plantas de menor altura $(83 \mathrm{~cm})$ se obtuvieron con los tratamientos testigo y $2 \mathrm{R} 3 \mathrm{~B}$.

Bergstrand et al. (2016) reportaron que la elongación del tallo es una respuesta del fitocromo, el cual se concentra en los meristemos apicales que son regiones donde se producen cambios drásticos en el desarrollo, una iluminación suplementada con luz roja aumenta la altura del crisantemo que como Lilium son plantas de día largo, pero el efecto es contrario en plantas de nochebuena (Euphorbia pulcherrima) que es planta de día corto (Dole y Wilkins, 2005) en las cuales se reduce la altura. El incremento en peso fresco y seco de las plantas bajo el tratamiento 4RB también correspondió con un mayor diámetro de tallo y de flor. Estos resultados concuerdan con lo que reporta Chen et al. (2018) que afirmaron que el mayor peso seco se obtiene bajo luz roja.

El máximo valor de área foliar se registró en el tratamiento R4B (tratamiento con 20\% de luz roja y $80 \%$ de luz azul), lo cual es acorde a lo obtenido por Chen et al. (2018) quienes encontraron que los receptores de luz azul (criptocromos y fototropinas) tienen efecto sobre el desarrollo del área foliar. En cuanto a los parámetros colorimétricos, se observa que el valor de $\mathrm{C}$ se incrementó con la proporción de luz azul, lo que dio como resultado tépalos de un color rosa más intenso; por el contrario, el mayor valor del L (que va de 0 para el color negro a 100 para el color blanco) se presentó en $4 \mathrm{RB}$ ocasionando tépalos más pálidos.

El color de las flores es una de las características más importantes en las plantas ornamentales ya que afecta su valor comercial, los colores de las flores de Lilium se derivan principalmente de antocianinas y carotenoides, las antocianinas son el pigmento principal en cultivares con flores color rosa y marrones mientras que los carotenoides lo son de cultivares con flores amarillas y anaranjadas, ambos, antocianinas y carotenoides contribuyen al color rojo (Kong et al., 2015).

Sólo hay una antocianina, la cianidina, en los pétalos de varios colores de flores que van del rosa claro, rosa, rojo obscuro y marrón, pero el contenido es diferente, la diferencia de tono se correlaciona positivamente con el contenido de antocianinas (Yamagishi et al., 2012). En rosa (Rosa hibrida), crisantemo y campanula (Campanula portenschlagiana), Ouzounis et al. (2014) encontraron que una alta proporción de luz azul incrementaba la concentración de antocianinas.

\section{Densidad estomática}

El máximo valor se registró en hojas basales de R4B (Figura 1) donde la tasa de fluencia (número de fotones absorbidos por unidad de superficie) fue menor $\left(35 \mu \mathrm{mol} \mathrm{m} \mathrm{m}^{-2} \mathrm{~s}^{-1}\right)$ que en los ápices de las plantas $\left(90 \mu \mathrm{mol} \mathrm{m} \mathrm{m}^{-2} \mathrm{~s}^{-1}\right)$. De acuerdo con Matthews et al. (2020) la apertura estomática es impulsada por dos distintas rutas: con luz roja se tiene el mecanismo que coordina el comportamiento estomático con la fotosíntesis y ocurre a altas tasas de fluencia; la respuesta específica a la luz azul se satura a bajas intensidades $\left(5\right.$ a $\left.10 \mu \mathrm{mol} \mathrm{m} \mathrm{m}^{-2} \mathrm{~s}^{-1}\right)$, es independiente de la fotosíntesis y provoca la apertura estomática por las mañanas cuando el espectro solar está enriquecido con longitudes de onda del azul. 


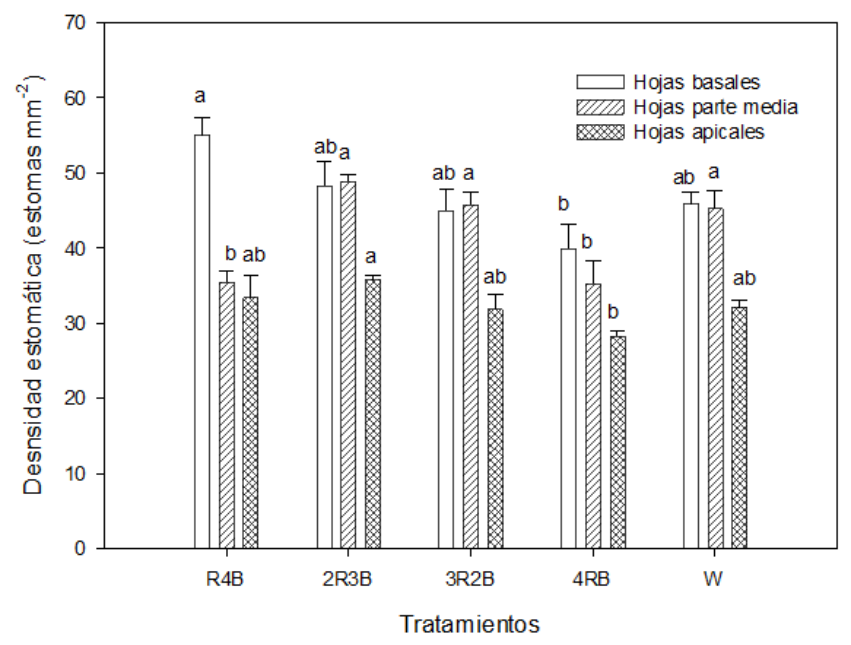

Figura 1. Efecto de la luz suplementaria LED roja, azul, blanca y sus combinaciones sobre la densidad estomática en hojas de Lillium spp. 'Corvara'. Medias con letras iguales no son diferentes (Tukey, $p \leq 0.05$ ); R: luz roja, B: azul y W: blanca; R4B: R 20\% + B 80\%, 2R3B: R 40\% + B $60 \%$, 3R2B: R 60\% + B 40\%, 4RB: R 80\% + B 20\% y W: 100\% de luz blanca.

\section{Pigmentos}

En la Figura 2 se observa que la mayor concentración de clorofila a, b, total y carotenoides antes de la floración y al momento del corte de los tallos se obtuvo en los tratamientos con mayor porcentaje de luz roja (4RB y $3 R 2 B$ ).
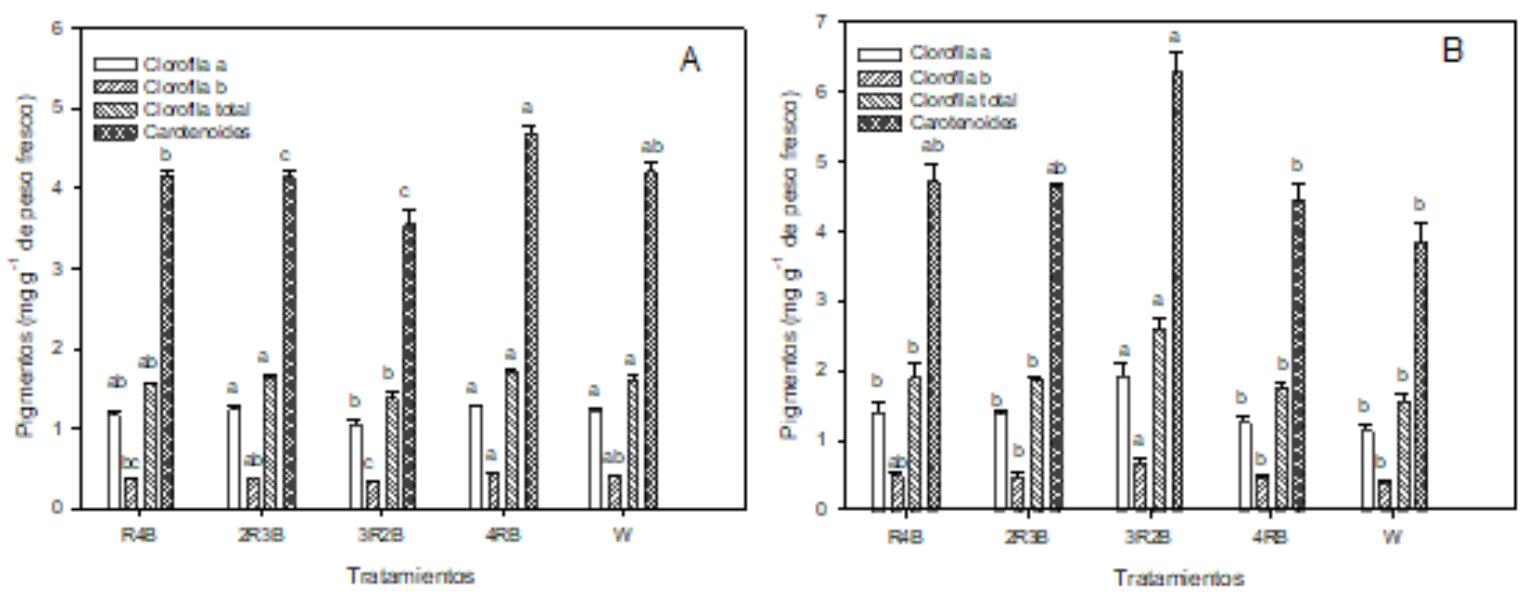

Figura 2. Efecto de la luz suplementaria LED roja, azul, blanca y sus combinaciones sobre la concentración foliar de pigmentos: A) antes de la floración; y B) después del corte de Lillium spp. 'Corvara'. Medias con letras iguales no son diferentes entre pigmentos (Tukey, $p \leq 0.05)$; R: luz roja, B: azul y W: blanca; R4B: R 20\% + B 80\%, 2R3B: R 40\% + B 60\%, 3R2B: R 60\% + B 40\%, 4RB: R 80\% + B 20\% y W: 100\% de luz blanca.

Folta y Childers (2008) indicaron que, aunque las clorofilas a y b tienen sus picos de absorción en el rango del azul y del rojo del espectro, el efecto de absorción de los distintos fotoreceptores se traslapa además de que bajo altas condiciones de luz los fitocromos, criptocromos y fototropinas 
se saturan, por lo que es difícil diferenciar el alcance de cada uno de ellos. Respecto a los demás pigmentos, en todos los tratamientos fue mayor la concentración de carotenoides, los cuales con un espectro de absorción entre 350 y $500 \mathrm{~nm}$ captan de manera eficiente mucha de la luz no absorbida por las clorofilas a y b para la fotosíntesis (Ouzounis et al., 2015). En rosa (Rosa hibrida) se encontró que la cantidad de carotenoides disminuyó en plantas cultivadas bajo luz azul (Bayat et al., 2018) lo cual concuerda con los resultados de este trabajo.

\section{Azúcares solubles totales}

En los tratamientos la concentración de azúcares de las hojas disminuyó en la floración, al momento de la cosecha se concentraron en el bulbo respecto a las hojas y raíces, ya que las escamas del bulbo al ser hojas modificadas almacenan agua y sustancias de reserva (Dole y Wilkins, 2005).

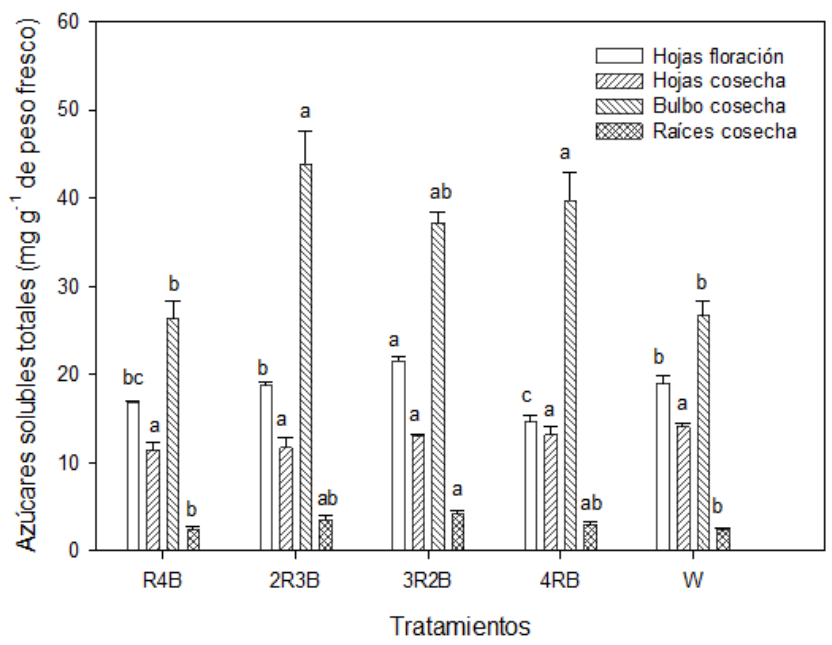

Figura 3. Efecto de la luz suplementaria LED roja, azul, blanca y sus combinaciones sobre la concentración de azúcares solubles totales de Lilium spp. 'Corvara'. Medias con letras iguales no son diferentes entre órganos (Tukey, $p \leq 0.05$ ); R: luz roja, B: azul y W: blanca; R4B: R $20 \%+$ B 80\%, 2R3B: R 40\% + B 60\%, 3R2B: R 60\% + B 40\%, 4RB: R 80\% + B 20\% y W: $100 \%$ de luz blanca.

En todos los tratamientos la concentración disminuye significativamente durante la floración porque la demanda de fotosintatos se acentúa cuando aparece el botón floral. Al momento de la cosecha los azúcares se concentran en el bulbo respecto a los demás órganos, ya que las escamas del bulbo al ser hojas modificadas almacenan agua y sustancias de reserva (Dole y Wilkins, 2005).

\section{Vida en florero y consumo de agua en poscosecha}

Las plantas bajo el tratamiento 3R2B acumularon más azúcares en las hojas para el periodo de floración (Figura 3), los cuales pudieron estar disponibles hasta postcosecha por lo que tuvieron una vida en florero más larga, lo que es una ventaja para su comercialización, aunque estadísticamente no es diferente a la vida en florero que se tuvo con el tratamiento 2R3B. En lisianthus (Eustoma grandiflorum), el contenido de azúcares favoreció el desarrollo del botón y la apertura floral, además de que aumentó la vida en florero (Cruz-Crespo et al., 2006). Las plantas con mayor vida en florero fueron las que consumieron más agua en postcosecha (Figura 4). 


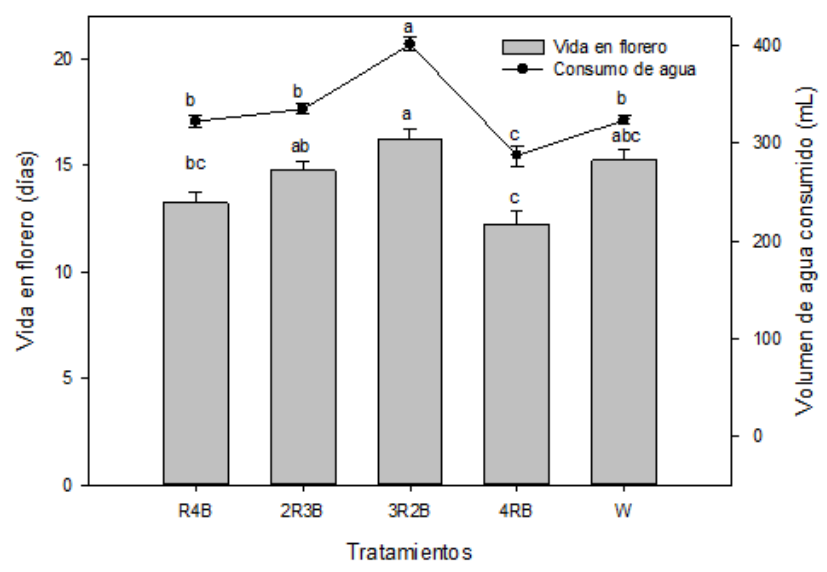

Figura 4. Efecto de la luz suplementaria LED roja, azul, blanca y sus combinaciones sobre la vida en florero y consumo de agua en postcosecha en plantas de Lilium spp. 'Corvara'. Medias con letras iguales no son diferentes entre órganos (Tukey, $p \leq 0.05$ ); R: luz roja, B: azul y W: blanca; R4B: R $20 \%$ + B 80\%, 2R3B: R 40\% + B 60\%, 3R2B: R 60\% + B 40\%, 4RB: R $80 \%$ + B $20 \%$ y W: $100 \%$ de luz blanca.

En ninguno de los tratamientos hubo caída o aborto de botones florales, ni se detectaron plagas o enfermedades, al contrario de lo que se encontró en plantas de tomate cultivadas sin luz azul, en las cuales se desarrollaron desórdenes foliares (Wollaeger y Runkle, 2013); asimismo, en lisianthus, la intensidad luminosa afecta la calidad de las flores, baja radiación luminosa causa aborto de flores, hojas amarillentas y decoloración de pétalos (Griesbach, 1992).

\section{Correlación lineal de Pearson}

Se detectaron entre las variables altura $v s$ peso seco de la parte aérea (0.89), altura $v s$ días a madurez de cosecha (0.74) y peso seco de la parte aérea $v s$ diámetro de flor $(0.87)$, por lo que existe una relación positiva entre las variables peso seco de la parte aérea, altura y diámetro de la flor.

\section{Conclusiones}

Las diferentes proporciones de luz roja y azul modificaron el crecimiento y desarrollo de Lillium spp. 'Corvara'. El tratamiento con $20 \%$ de luz roja $+80 \%$ de luz azul favoreció el desarrollo del área foliar y el color de pétalos más intenso, el ciclo de cultivo más corto se obtuvo aplicando $40 \%$ de luz roja $+60 \%$ de luz azul; la vida en florero más larga se tuvo en las plantas cultivadas con $60 \%$ de luz roja $+40 \%$ de azul, mientras que, con $80 \%$ de luz roja $+20 \%$ de luz azul se tuvieron las flores de mayor diámetro y las plantas de mayor altura.

\section{Literatura citada}

AOAC. 1980. Association of Official Analytical Chemists. Official Methods of Analysis. $12^{\text {th }}$ (Ed.). Association of Official Analytical Chemists. Washington DC, USA. 1018 p.

Bayat, L.; Arab, M.; Aliniaeifard, S.; Seif, M.; Lastochkina, O. and Li, T. 2018. Effects of growth under different light spectra on the subsequent high light tolerance in rose plants. Ann. Bot Comp. Plants. 10(5):1-17. https://doi.org/10.1093/aobpla/ply052. 
Bergstrand, K. J.; Asp, H. and Schussler, H. K. 2016. Grow control of ornamental and bedding plants by manipulation of photoperiod and light quality. Acta Hortic. 11(34):33-39. https://doi.org/10.17660/ActaHortic.2016.1134.5.

Cruz-Crespo, E.; Arévalo-Galarza, L.; Cano-Medrano, R. y Gaytán-Acuña, E. A. 2006. Soluciones pulso en la calidad postcosecha de lisianthus (Eustoma grandiflorum Raf.). Agric. Téc. Méx. 32(2):191-200.

Chen, L.; Xue, X.; Yang, Y.; Chen, F.; Zhao, J.; Wang, X.; Khan, A. and Hu, Y. 2018. Effects of red and blue LEDs on in vitro growth and microtuberization of potato single-node cuttings. Frontiers Agr. Sci. Eng. 5(2):197-205. http://journal.hep.com.cn/fase.

Darko, E.; Heydarizdeh, P.; Schoefs, B. and Sabzalian, M. R. 2014. Photosynthesis under artificial light: the shift in primary and secondary metabolism. Philosophical Transactions of the Royal Society Botany. 369(3)1-7. https://doi.org/10.1098/rstb.2013.0243.

Deram, P.; Lesfurd, M. G. and Orsat, V. 2014. Supplemental lighting orientation and red to blue ratio of light-emitting diodes for greenhouse tomato production. Hortscience. 49(4):448452. https://doi.org/10.212737hortsci.49.4.448.

Dole, M. J. and Wilkins, F. H. 2005. Floriculture principles and species. $2^{\mathrm{a}}$. Edition. Prentice Hall. Upper Sadle River, New Jersey, USA. 1023 p.

Fantini, E.; Sulli, M.; Aprea, G.; Jiménez-Gómez, J.; Bendahmane, A.; Perrotta, G.; Giuliano, G. and Facella, P. 2019. Pivotal roles of cryptochromes 1a and 2 in tomato development and physiology. Plant Physiol. 179(2):732-748. www.plantphysiol.org/cgi/doi/10.1104/ pp.18.00793.

Folta, K. M. and Childers, K. S. 2008. Light as a grow regulator: controlling plant biology with narrow bandwidth solid-state lighting systems. HortScience. 43(7):1957-1963. https://doi.org/10.21273/hortsci.43.7.1957.

García, V. R. y Companioni, G. B. 2018. Lilium: situación actual en México. Revista de Economía y Sociedad de México. 23 p. https://www.eumed.net/rev/tecsistecatl/n23/lilium.html.

Griesbach, R. J. 1992. Correlation of $\mathrm{pH}$ and light intensity on flower color in potted Eustoma grandiflorum Grise. Sci. Hortic. 27(7):817-818.

Jakopic, J.; Veberic, R.; Stampar, F. 2007. The effect of reflective foil and hail nets on the lighting, color and anthocyanins of 'Fuji' apple. Sci. Hortic. 115(1)40-46. https://doi.org/10.1016/j.scienta.2007.07.014.

Kong, Y.; Dou, X. Y.; Bao, F.; lang, L. X. and Bai, J. R. 2015. Advances in flower color mechanism of Lilium. Acta Hort Sinica. 42(9):1747-1759. https://doi.org/10.16420/j.issn.0513353x.2015-0103.

Matthews, J. S. A.; Vialet-Chabrand, S. and Lawson T. 2020. Role of blue and red light in stomatal dynamic behavior. J. Exp. Bot. 71(7):2253-2269. https://doi.org/10.1093/jxb/erz563.

Morrow, R. C. 2008. LED lighting in horticulture. HortScience. 43(7):1947-1950. https://doi.org/10.21273/HORTSCI.43.7.1947.

Meng, Q. and Runkle, E. S. 2016. Moderate intensity blue radiation can regulate flowering, but not extension growth, of several photoperiodic ornamental crops. Environ. Exp. Bot. https://doi.org/10.1016/j.envexpbot.2016.10.006.

Ojodeagua, A. J. L.; Castellanos, R. J. Z.; Muñoz, R. J. J.; Alcántar, G. G.; Tijerina, C. L.; Vargas, T. P.; Enríquez, R. S. 2008. Eficiencia de suelo y tezontle en sistemas de producción de tomate en invernadero. Rev. Fitotec. Mex. 31(4):367-374.

Olschowski, S.; Geiger, E. M.; Herrmann, J. V.; Sander, G. and Gruneberg, H. 2016. Effects of red, blue and white LED irradiation on root and shoot development of Calibrachoa cuttings in comparison to high pressure sodium lamps. Acta Hortic. 1134:245-250. https://doi.org/10.17660/ActaHortic.2016.1134.33. 
Ouzounis, T.; Fretté, X.; Rosenqvist, E. and Ottosen, C. O. 2014. Spectral effects of supplementary lighting on the secondary metabolites in roses, chrysanthemums, and campanulas. J. Plant Physiol. 171(16):1491-1499. https://doi.org/10.1016/j.jplph.2014.06.012.

Ouzounis, T.; Rosenqvist, E. and Ottosen, C. O. 2015. Spectral effects of artificial light on plant physiology and secondary metabolism: a review. HortScience. 50(8):1128-1135. https://doi.org/10.21273/HORTSCI.50.8.1128.

Randall, W. C. and Lopez, R. G. 2014. Comparison of supplemental lighting from high pressure sodium lamps and light emitting-diodes during bedding plant seedling production. Hortscience. 49(5):589-595, https://doi.org/10.21273/hortsci.49.5.589.

SAS. 2002. Institute Inc. SAS/STAT Guide for personal computers. Versión 9. SAS Institute North Caroline. $890 \mathrm{p}$.

SMN. 2021. Servicio Meteorológico Nacional. Normales climatológicas del Estado de México, Estación 15170 Chapingo. https://smn.conagua.gob.mx.

SIAP. 2020. Servicio de Información Agroalimentaria y Pesquera. Anuario estadístico de la producción agrícola. Secretaría de Agricultura y Desarrollo Rural. Ciudad de México. www.gob.mx/siap.

Steiner, A. A. 1961. A universal method for preparing nutrient solutions of a certain desired composition. Plant Soil. 15(2):134-154.

Witham, F. H.; Blaydes, D. F. and Devlin, R. M. 1971. Experiments in plant physiology. Van Nostrand Reinhold Company. New York, USA. 245 p.

Wollaeger, H. M. and Runkle, E. S. 2013. Growth responses of ornamental annual seedlings under different wavelengths of red light provide by light-emitting diodes. HortScience. 48(12):1478-1483. https://doi.org/10.21273/HORTSCI.48.12.1478.

Xie, B.; Wei, J.; Zhang, Y.; Song, S.; Su, W.; Sun, G.; Hao, Y. and Liu, H. 2019. Supplemental blue and red light promotes lycopene synthesis in tomato fruits. J. Integr. Agric. 18(3):590598. https://doi.org/10.1016/52095-3119(18)62062-3.

Yamagishi, M.; Yoshida, Y. and Nakayama, M. 2012. The transcription factor LhMYB12 determines anthocyanin pigmentation in hybrid lilies (Lilium spp.) and regulates pigment quantity. Mol. Breed. 30(2):913-925. https://doi.org/10.1007/s11032-011-9675-6. 